\title{
Who cares for hospital dentists?
}

\author{
Peter Dyer, Responsible Officer, University Hospitals of Morecambe Bay NHS FT; lan Oakley, Policy Officer BDA and \\ Pete Brotherton -Associate Specialist in Oral and Maxillo-Facial Surgery Hull and East Yorkshire NHS Trust
}

The BDJ Upfront section includes editorials, letters, news, book reviews and interviews. Please direct your correspondence to the News Editor,

Adrian O'Dowd at BDJNews@nature.com. Press releases or articles may be edited, and should include a colour photograph if possible.

$\mathrm{T}$ he year 2017 marked a number of fiftieth anniversaries. It was 50 years since the Beatles released their album Sgt Pepper's Lonely Hearts Club Band; since the first Ford car was introduced into Britain; since the BDA's headquarters were opened by Her Majesty the Queen and 50 years since the BDA's current Central Committee for Hospital Dental Services (CCHDS) came into existence. Hospital dentistry's need for representation had in fact been recognised even earlier with the establishment of the BDA's Hospital Groups in 1947, to provide representation for the Hospital Dentistry that was emerging hand in hand with the establishment of the NHS in 1948. To mark the fiftieth anniversary of the creation of CCHDS the Committee decided to survey BDA hospital dental staff. This has provided us with information that we are not aware any other body holds and so gives us a unique insight into the hospital dental service.

The CCHDS continues to work with BDA primary care committees at the BDA to ensure that there is a rational implementation of policies that support hospital dentists and their general dental practitioner (GDP) colleagues to provide the best service for their patients. However, many hospital dentists are working in an increasingly restrictive environment, which is challenging the relationship with GDPs. An environment in which the traditional letter between GDPs and hospital is now redirected to a third party who makes an assessment as to the 'appropriateness' of the referral. One in which extra patients are randomly squeezed onto already heavily booked clinics to avoid a breach in waiting time targets, and an environment where 'prior approval' may be sought from a commissioning panel before a treatment can be undertaken.

Those patients not meeting the referral criteria for a hospital appointment may find themselves travelling to a Tier 2 dentist for treatment. This pattern for patient management will only be successful if the pathways have been designed by the local clinicians (both hospital and dental practitioners), working in conjunction to achieve the best outcomes. The Tier 2 dentists need to be supported by their hospital colleagues in a network in which there are opportunities to meet for case discussions. Adequate funding, with time set aside in the working week, must be in place.

Similarly, Getting it Right First Time (GIRFT), an initiative that aims to improve the quality of outcome and patient experience, enhance safety, and address unacceptable variation in practice also has the potential to put distance between the dentist and their hospital service. Whilst welcoming the aims, CCHDS has concerns that integrated care consultants and SAS grades (the highest ratio in any medical speciality) with a lot of oral surgery not being coded to those doing the work. The BDA's hospital dental survey has looked at what its hospital members are doing in their job and their mood, and the results are published in this issue. ${ }^{1}$

Unfortunately (and not surprisingly) for those that work as a SAS grade their responses showed low morale and a perceived poor potential to develop their careers. In fact, the responses for morale rank lower than those working as primary care associates under the current UDA system, which in itself, must say something! CCHDS and the wider BDA has listened to the feedback from its membership and it is informing its ongoing strategy.

\section{'Many hospital dentists are now working in an increasingly restrictive environment, which is challenging the relationship with GDPs...'}

systems looking to manage their budgets could amalgamate local units onto one geographically remote site using GIRFT as the instrument.

The CCHDS also continues to work with the secondary care practitioners of all grades. In the year of the seventieth anniversary of the NHS is interesting to note that it is almost 60 years since the first mention of the associate specialist grade in the NHS. Now collectively termed SAS grades (for staff Grades, associate specialists and speciality doctor grades) the BDA looks after these grades and how their role is evolving. We are keen to remind clinicians that these grades (often incorrectly termed 'middle grades' - there is in fact no such grade in NHS employment) deliver significant care for our population. For instance, in oral and maxillofacial surgery (OMFS) there are almost equal numbers of OMFS
A recent letter from a specialist society (BAOS) has sparked a unique meeting amongst multiple stakeholders (again a BDA first) looking at disparities for recognition of training between medicine and dentistry within the regulatory bodies and the CCHDS will host this meeting in the autumn; we will keep BDA members posted.

While the Hospital Dental Service has changed hugely from 1967, let alone 1947, some things are constant. As the history of the BDA records, in the 1950s hospital dentists were upset by the failure of the then government to act when faced with recommendations for pay increases for hospital staff. So as the present government's response to the 2018 DDRB announcement shows, some things never change.

DOI: 10.1038/sj.bdj.2018.817 